trode surface processes, and, more recently, even an atomic-level view of the electrode surface; spectroscopic methods are dismissed in a single paragraph and other techniques, such as scanning tunnelling microscopy, are not mentioned.

Overall then, if one is looking for a good treatment of classical physical electrochemistry and its traditional topics, this book is a good alternative to several others of a similar type. Unfortunately, the usefulness of the volume is marred by a total lack of references in the text to any of the original literature (there is, however, a bibliography of general monographs and reviews). Readers with a good background in physics and physical chemistry will probably prefer a more chemical approach with greater attention to modern methods.

Allen J. Bard is in the Department of Chemistry, University of Texas, Austin, Texas 78712, USA.

\section{Chance and necessity}

\section{David Goodstein}

The Refrigerator and the Universe: Understanding the Laws of Energy. By Martin Goldstein and Inge F. Goldstein. Harvard University Press: 1993. Pp. 433. $\$ 29.95$, £23.95.

IN a world rapidly running out of fossil fuel, the second law of thermodynamics may well turn out to be the central scientific truth of the twenty-first century. And yet, even though it was discovered 150

\section{IMAGE UNAVAILABLE FOR COPYRIGHT REASONS}

years ago, it is well and truly understood by only the tiniest handful of the globe's five billion inhabitants. Clearly, there is much need to spread the word. We have here an attempt to do that.

The Refrigerator and the Universe certainly has its heart in the right place, to say nothing of its nouns and verbs. Its title brings to mind the fact that the second law, discovered by Nicolas Carnot while contemplating a sooty, clanging steam engine, applies even to black holes. Shouldn't an epic story like that be worth a great book? The answer is yes, and I have long thought a book telling it was badly needed. But this, alas, is not the one.

The Goldsteins really really want to teach us thermodynamics so that we can marvel with them at all its beautiful subtleties and consequences. The trouble is that before we can really really understand thermodynamics, we first have to understand a few other things, such as mechanics, energy, kinetic theory, statistics .... And so we have to wade through pages and pages of work and force, how energy and its conservation were discovered, bouncing molecules and Brownian movement, and flipping coins and shuffling decks, to say nothing of caloric theory and how Carnot's arguments were snatched from its grips, allow-

? ing the concept of entropy to emerge. All of this the Goldsteins tell in clear but stiff $\sum_{\Sigma}^{\frac{\pi}{5}}$ and uninspired prose, using simple algebraic equations whenever they deem them necessary. There are even mathematical appendices so readers can brush up on units and logs and exponentials. If, pace Stephen Hawking, every equation in a popular science book reduces the audience by a half, then I should be the only one that reads this book.

The story is told historically for the most part, although history isn't quite the right word for it. Perhaps hagiography is closer. We are told the tale of one scientific saint after another in a style that is depressingly familiar from high-school and college science textbooks. In fact, the real surprise about this book is that it has not been packaged as a textbook. All it needs is a few problems and discussion items at the end of each chapter and it would serve admirably in a course titled "Thermodynamics for Poets". (Do any such courses exist?)
The dust jacket promises that "The Refrigerator and the Universe will appeal to those with little scientific background, but a curiosity about the concepts of energy and entropy, as well as to scientists who use thermodynamics in their research but would like to broaden their understanding of the subject." I am afraid I disagree at both ends of the scale. There are just too many hundreds of pages of plodding before anything interesting happens. To be sure, one really does have to grasp all the preliminaries before one can master the second law, but to get the attention of either the casual reader or the uninformed scientist, that part of the story has to be told with enough wit and insight to make it appealing in its own right. Instead, this book presents readers not with exciting reading, but with medicine they ought to take because it's good for them. I'm afraid that's not good enough to help to solve the worldwide shortage of people who understand the second law. The Goldsteins have made a noble and stout-hearted attempt, but victory over ignorance will have to await another day, and another book.

David Goodstein is in the Department of Physics, California Institute of Technology, Pasadena, California 91125, USA. 